\title{
Comprehensive Analysis of the Prognosis for Chemokine-Like Factor MARVEL Transmembrane Domain-Containing Family in Hepatocellular Carcinoma
}

\section{Dan Li}

Nanchang University Second Affiliated Hospital

Shenglan Huang

Nanchang University Second Affiliated Hospital

Liying Sun

Nanchang University Second Affiliated Hospital

Jianbing Wu ( $\sim$ hhgwjb@163.com )

Nanchang University Second Affiliated Hospital https://orcid.org/0000-0002-4576-7286

\section{Research Article}

Keywords: CMTM, hepatocellular carcinoma, DNA methylation, prognosis, biomarkers

Posted Date: August 3rd, 2021

DOl: https://doi.org/10.21203/rs.3.rs-749291/v1

License: (c) (1) This work is licensed under a Creative Commons Attribution 4.0 International License.

Read Full License 


\section{Abstract}

Background and Purpose: Chemokine-like factor (CKLF) MARVEL transmembrane domain-containing family (CMTMs), including chemokine-like factor (CKLF) and CMTM1 to CMTM8. All of these genes participate in tumor development and metastasis of hepatocellular carcinoma (HCC). However, prognosis values and the distinct roles of different CMTMs in HCC are still un-clarified.

Materials and Methods: To address this issue, the study explored the function of CMTM family members in the prognosis of HCC by TCGA dataset, UALCAN, Kaplan-Meier Plotter, MethSurv, TIMER2.0 databases.

Results: The CKLF and CMTM1/3/4/7/8 mRNA expression was obviously elevated in HCC tissues, while CMTM2/5/6 was lowly expressed. Besides, the mRNA level of CMTMs was closely linked to cancer stage, node metastasis status and tumor grades in HCC patients. What's more, the expression of CMTM2/5 was correlated with favorable overall survival (OS), while high expressions CKLF and CMTM1/7 were associated with poor OS. Simultaneously, low CKLF and CMTM1/4/7 expressions were significantly relevant to better progression-free survival (PFS), while low CMTM5 expression was apparently concerned with worse PFS. In DNA methylation level, 47 CpGs of CMTMs displayed markedly prognostic values. Multivariate cox analysis also revealed that affecting shorter OS outcomes prognostic factors of CKLF and CMTM7 were independent. GSEA results suggested that CKLF and CMTM7 participated in the development and progression of HCC accompanied with multiple biological processes. Furthermore, CMTMs were dramatically associated with tumor immune infiltrating cells (TIICs) (e.g., neutrophils cells), but CMTM8 expression level was no significant correlations with TIICs.

Conclusion: This study renders new insight into the prognostic values of CMTM family members in HCC. Overall, our results indicated that CKLF and CMTM7 could be the prognostic biomarkers in HCC patients.

\section{Introduction}

Hepatocellular carcinoma which occupied $75 \%-85 \%$ of liver cancer and the mortality rate is high[1]. HCC is generally diagnosed in the middle or late stage. Despite advances in medical technology for the treatment of HCC, long-term survival remains low. The reason is mainly due to high recurrence rates and early metastasis and this is a major barrier for HCC treatment[2,3]. Therefore, the identification of reliable biomarkers for the prognostic prediction of HCC is crucial for individualized treatment.

Chemokine-like factor MARVEL transmembrane domain-containing family (CMTMs) consists of CKLF, CMTM1, CMTM2, CMTM3, CMTM4, CMTM5, CMTM6, CMTM7 and CMTM8 and the sites of these genes are various[4]. CKLF and CMTM1-4 reside in chromosome 16q22. CMTM5 was located on chromosome 14q11.2 and CMTM6-8 lies in chromosome 3p23[5]. Up to now, studies showed that CMTM family members do not only have significant effects on immune, circulatory and male reproductive systems[68] but also closely act in the development of cell proliferation, apoptosis, metastasis, and immune evasion $[9,10]$. For instance, it has been confirmed that CKLF was related to vascular invasion and highly expressed in HCC. Mechanistically, CKLF could trigger the signaling pathway of IL-6/STAT3 to promote 
HCC proliferation and migration and inhibit the apoptosis induced by Doxorubicin[11]. Research showed that over-expression of CMTM2 predicted a better prognosis of diffuse-type gastric cancer and CMTM5 has been indicated to be an adverse expectation factor in breast cancer[12]. Besides, CMTM8 was downregulated in bladder cancer, and the bladder cancer tissue cell growth migration and invasion was apparently inhibited after overexpression of CMTM8. Thus, we speculate that CMTM8 was a statistically significant predictor of better OS and PFS in bladder cancer patients[13].

However, the prognostic values of the CMTM in HCC have not been thoroughly explored, and the roles of distinct CMTM family genes in the progress of HCC have not been well investigated. In this study, we comprehensively analyzed the level and DNA methylation of distinct CMTMs and their correlation with clinical parameters and prognosis in HCC patients. Furthermore, we also evaluated the expression of CMTM and immune cell infiltration levels in HCC which have inter-relationships. Besides, we also investigate the potential functions underlying the involvement of CMTMs in the carcinogenesis of HCC.

\section{Materials And Methods}

\section{The mRNA expression of CMTM family members in TCGA database}

The mRNA expression of CMTMs in HCC was evaluated by The Cancer Genome Atlas (TCGA: https://cancergenome.nih.gov/) database. The data from the TCGA portal was analyzed and utilized the limma package in R 3.6.1software. Using the pheatmap package to visualize the results.

Clinicopathological characteristics analysis of CMTM family members via UALCAN database

Through the UALCAN (http://ualcan.path.uab.edu/) database, we investigate the mRNA expression of CMTMs in HCC and their relationship with clinicopathological parameters, including cancer stages and node metastasis status. $p<0.05$ indicated statistical significance.

\section{Survival Analysis Of Cmtm Family Members In Kaplan- meier Plotter}

The prognostic values of each CMTM family genes to overall survival (OS) and progression-free survival (PFS) in HCC patients were assessed by Kaplan-Meier plotter (http://kmplot.com/analysis/). The prognostic KM survival plots were displayed on hazard ratio (HR), $95 \%$ confidence intervals (Cls) and logrank $p$-value. $p<0.05$ represented statistical significance.

DNA methylation data and Prognostic roles of CMTM family members in MethSurv

The DNA methylation date of CMTM family genes in TCGA-LIHC was extracted from MethSurv (https://biit.cs.ut.ee/methsurv/) database. The corresponding prognostic value of different CpG 
methylation sites of CMTM family genes in patients with HCC was explored.

\section{Prognostic Values Of Cmtm Family Members}

Through the samples of $374 \mathrm{HCC}$ and 50 matched control samples, the transcriptome information and corresponding clinical data for HCC were downloaded from TCGA-LIHC to investigate the effect of CMTM family genes. We screened this information and analyzed the remaining 351 tumor samples after the deletion of incomplete clinical information to investigate statistical associations between CMTM family genes and various clinicopathological features. The study was obtained using the survival and survminer package in R3.6.1 software. Univariate and multivariate Cox analyses were conducted to establish a proportional risk regression model. $p<0.05$ was considered statistically significant.

Correlation between Tumor immune infiltrating cells (TIICs) and CMTM family members

Tumor immune Estimation Resource 2.0 (TIMER2.0 http://timer.cistrome.org) database contains various kinds of tumor immune cell infiltration data. The relationship between tumor immune infiltrating cells (TIICs) and members of the CMTM family was explored and utilized by TIMER 2.0 platform. In TIMER 2.0, the TIICs include CD8 ${ }^{+}$T-cells, CD $4^{+}$T-cells, B-cells, neutrophils, macrophages and dendritic cells. Tumor purity was performed for the correction of the Spearman correlation analysis. Statistically, $p<0.05$ was considered statistically significant.

\section{Gene Set Enrichment Analysis}

The CMTM family differential gene expression data were imported into GSEA (version 4.0.1; http://software.broadinstitute.org/gsea/index.jsp) to further explore CMTM family genes in the pathways and potential molecular mechanism underlying of HCC. Each analysis was performed 1000 times using weighted enrichment statistics and false discovery rate $($ FDR $)<0.05$ were identified as significantly rich pathways.

\section{Results}

\section{The mRNA expression of CMTM family genes in HCC}

Based on the TCGA database, the CMTM family members in HCC were detected. Interestingly, compared with the adjacent non-tumor samples, the mRNA expression of CKLF and CMTM1/3/4/7/8 was significantly up-regulated in HCC, whereas the CMTM2/5/6 expressions were significantly downregulated (Fig. 1A). Furthermore, the heat maps were used to visualize the differential expression of CMTM family members (Fig. 1B).

Relationship between the mRNA expression of different CMTM family genes and clinicopathological parameters in patients with HCC 
The UALCAN database was used to determine the mRNA expression of each CMTM member in HCC and to analyze the relationship such as cancer stage, node metastasis status and tumor grade between different CMTM mRNA expression and clinicopathological parameters in HCC patients. As shown in Fig. 2, CMTMs were dramatically correlated with cancer stages in HCC patients, and patients who have higher mRNA expression of CMTMs with late stage. The highest level of CKLF and CMTM2/6 were displayed in stage 4 (Fig. 2A, C, G), while the highest CKLF and CMTM1/3/4/5/7/8 were displayed in stage 3 (Fig. 2A, B, D-F, H, I). As Fig. 3 shows, most CMTM family members were associated with nodal metastasis for HCC patients, except for CMTM2. Furthermore, just like Fig. 4, the CKLF and CMTM1/7 were highest expressed in tumor grade 4 (Fig. 4A, B, H), while CKLF and CMTM1/3/4/7/8 were the highest expressed in grade 3 (Fig. 4A, B, D, E, H, I). In brief, the results figured out that mRNA expressions of 9 CMTM family members were apparently associated with the progression in HCC patients.

\section{Prognostic Value Of Cmtm Family Genes In Hcc Patients}

We investigated the relationship between CMTMs mRNA expression and survival time in HCC patients. The prognostic value of each CMTM family genes were determined by Kaplan-Meier plotter. KM curve and log-rank test analysis demonstrated that patients with higher CKLF and CMTM1/7 mRNA expression and poor OS were significantly correlated,while higher CMTM2/5 mRNA expression prompted a better OS $(p<0.05$, Fig. 5A). Additionally, increased mRNA levels of CKLF/CMTM1/7 or decreased level of CMTM5 was correlated with worse PFS. They are all considered to be statistically significantly correlated $(p<0.05$, Fig. 5B).

\section{Prognostic values of CMTM family genes DNA methylation in HCC}

All the DNA methylation levels of CMTM family proteins with the prognostic values of every single CpGsite were displayed. In fact, cg01434228 of CKLF, cg07933092 of CMTM1, cg05976575 of CMTM2, cg03265991 of CMTM3, cg08571905 of CMTM4, cg16290720 of CMTM5, cg21331107 of CMTM6, cg11360860 of CMTM7 and cg03927025 of CMTM8 showed the highest DNA methylation (Fig. 6). Then, 6 CpGs of CKLF, 9 CpGs of CMTM1, 1 CpG of CMTM2, 3 CpGs of CMTM3, 8 CpGs of CMTM4, 2 CpGs of CMTM5, 4 CpGs of CMTM6, 10 CpGs of CMTM7 and 4 CpGs of CMTM8 were associated with significant prognosis of HCC patients $(p<0.05$, Table 1$)$. 
Table 1

The significantly prognostic values of CpG in the CMTM family members $(p<0.05)$

\begin{tabular}{|c|c|c|}
\hline Gene-CpG & HR & LR test pvalue \\
\hline CKLF-Body-S_Shelf-cg01434228 & 1.733 & 0.0069 \\
\hline CKLF-Body-S_Shore-cg00004055 & 1.443 & 0.041 \\
\hline CKLF-1 stExon;5'UTR-Island-cg10200312 & 1.770 & 0.006 \\
\hline CKLF-1stExon;5'UTR-Island-cg12513308 & 0.683 & 0.042 \\
\hline CKLF-1 stExon-Island-cg06014631 & 1.563 & 0.018 \\
\hline CKLF-TSS1500-N_Shore-cg08287306 & 1.706 & 0.023 \\
\hline CMTM1-Body-N_Shelf-cg26614624 & 1.744 & 0.0016 \\
\hline CMTM1-Body-S_Shelf-cg09839874 & 0.591 & 0.0038 \\
\hline CMTM1-Body-N_Shore-cg05054953 & 1.654 & 0.0077 \\
\hline CMTM1-TSS200-Island-cg09106154 & 1.695 & 0.0047 \\
\hline CMTM1-TSS200-Island-cg02053054 & 1.741 & 0.0085 \\
\hline CMTM1-TSS200-Island-cg04791678 & 1.495 & 0.022 \\
\hline CMTM1-1stExon;5'UTR; TSS200-Island-cg07933092 & 1.951 & 0.0018 \\
\hline CMTM1-Body;1 stExon-Island-cg15691822 & 2.235 & 0.00024 \\
\hline CMTM1-Body;1 stExon-S_Shore-cg00939649 & 1.912 & 0.00033 \\
\hline CMTM2-Body-S_Shore-cg27159096 & 0.645 & 0.013 \\
\hline CMTM3-3'UTR-Open_Sea-cg03265991 & 0.589 & 0.0039 \\
\hline CMTM3-Body-S_Shore-cg06251289 & 0.661 & 0.026 \\
\hline CMTM3-5'UTR;1 stExon-Island-cg03860859 & 2.014 & 0.0013 \\
\hline CMTM4-TSS200-Island-cg14395546 & 2.274 & $4 \mathrm{e}-04$ \\
\hline CMTM4-TSS1500-Island-cg01862266 & 1.420 & 0.047 \\
\hline CMTM4-TSS1500-Island-cg10021295 & 1.457 & 0.032 \\
\hline CMTM4-1stExon-Island-cg10051620 & 1.693 & 0.012 \\
\hline CMTM4- Body-Open_Sea-cg08571905 & 1.587 & 0.011 \\
\hline CMTM4-Body-Open_Sea-cg07849409 & 1.600 & 0.0085 \\
\hline CMTM4-3'UTR-Open_Sea-cg04394390 & 0.648 & 0.015 \\
\hline CMTM4-TSS1500-S_Shore-cg18693704 & 0.580 & 0.0036 \\
\hline
\end{tabular}




\begin{tabular}{|lll|}
\hline Gene-CpG & HR & LR test pvalue \\
\hline CMTM5-1stExon-Open_Sea-cg00174500 & 1.451 & 0.042 \\
\hline CMTM5-TSS200-Open_Sea-cg01747222 & 1.705 & 0.0098 \\
\hline CMTM6-Body-N_Shore-cg26280526 & 0.644 & 0.019 \\
\hline CMTM6-Body-Island-cg27109514 & 1.529 & 0.033 \\
\hline CMTM6-Body-N_Shelf-cg21331107 & 1.990 & $9.9 \mathrm{e}-05$ \\
\hline CMTM6-3'UTR-Open_Sea-cg06296151 & 1.927 & 0.00044 \\
\hline CMTM7-Body-Open_Sea-cg11360860 & 0.655 & 0.018 \\
\hline CMTM7-Body-Open_Sea-cg07116972 & 0.571 & 0.0023 \\
\hline CMTM7-TSS200-N_Shore-cg13808147 & 1.538 & 0.014 \\
\hline CMTM7-Body-N_Shore-cg00797278 & 1.857 & 0.00073 \\
\hline CMTM7-Body-N_Shore-cg20168479 & 1.908 & 0.0011 \\
\hline CMTM7-Body-Island-cg06175243 & 0.567 & 0.0025 \\
\hline CMTM7-Body-Island-cg10163776 & 1.877 & 0.0032 \\
\hline CMTM7-TSS200-Island-cg16154073 & 1.544 & 0.015 \\
\hline CMTM7-1stExon;5'UTR-Island-cg07881061 & 1.577 & 0.021 \\
\hline CMTM7-Body-S_Shore-cg16277214 & 0.6000 & 0.014 \\
\hline CMTM8-Body-Open_Sea-cg03927025 & 1.611 & 0.027 \\
\hline CMTM8-Body-Open_Sea-cg24924051 & 1.480 & 0.026 \\
\hline CMTM8-TSS1500-Island-cg02059034 & 1.648 & 0.0043 \\
\hline CMTM8-1stExon-Island-cg10553028 & & \\
\hline
\end{tabular}

Independent prognostic values of CMTM family genes in terms of survival outcome in patients with HCC

We extracted transcriptomic dates (374 HCC tumor samples and 50 adjacent non-tumor samples) and corresponding clinical information from $351 \mathrm{HCC}$ patients containing complete clinicopathological parameters. Univariate and multivariate COX analyses were performed according to the mRNA expression of CMTM family proteins, combined with age, sex, tumor stage and tumor grade of tumor patients. Univariate Cox analysis showed that high CKLF/CMTM7 expression and tumor stage were associated with poor prognosis in $\mathrm{HCC}$ patients (Table 2). Through the multivariate COX analysis of these factors and the prognosis of HCC patients, CKLF/CMTM7 mRNA expression and tumor stage were deemed as independent risk factors for the HCC patients' shorter survival time (Fig. 7A and B). Collectively, the results 
indicated that mRNA expression of CKLF and CMTM7 were determined as independent prognostic factors for HCC patients in OS results.

Table 2

Univariate Cox proportional hazards regression analyses of CMTM family genes and clinicopathological features in HCC

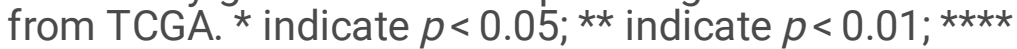
indicate $p<0.001$.

\begin{tabular}{|llll|}
\hline Parameter & \multicolumn{4}{l|}{ Univariate analysis } \\
\hline & Hazard ratio & $95 \% \mathrm{Cl}$ & $P$ value \\
\hline Age & 1.01 & $0.996-1.025$ & 0.171 \\
Gender & 0.816 & $0.552-1.206$ & 0.307 \\
\hline Grade & 1.116 & $0.863-1.442$ & 0.403 \\
\hline Stage & 1.672 & $1.354-2.064$ & $1.77 \mathrm{E}-06^{\star \star \star *}$ \\
\hline CKLF & 1.045 & $1.015-1.075$ & $0.003^{\star \star}$ \\
\hline CMTM1 & 2.509 & $0.849-7.412$ & 0.096 \\
\hline CMTM2 & 1.369 & $0.802-2.336$ & 0.249 \\
\hline CMTM3 & 1.014 & $0.994-1.034$ & 0.175 \\
\hline CMTM4 & 1.017 & $0.94-1.101$ & 0.669 \\
\hline CMTM5 & 0.018 & $0-503.087$ & 0.442 \\
\hline CMTM6 & 1.015 & $0.995-1.036$ & 0.133 \\
\hline CMTM7 & 1.132 & $1.052-1.218$ & $0.001^{\star *}$ \\
\hline CMTM8 & 0.998 & $0.981-1.016$ & 0.862 \\
\hline
\end{tabular}

\section{Correlation between tumor immune infiltrating cells (TIICs) and CMTM family genes}

We further investigated the relationship between TIICs and CMTM family members (Fig. 8).

CMTM $1 / 2 / 3 / 5 / 6 / 7$ were markedly negatively associated with tumor purity $(p<0.05)$, revealing that CMTM1/2/3/5/6/7 were highly expressed in the HCC microenvironment. Additionally, as shown in the scatter plots, CKLF and CMTM1/3/6/7 in HCC was positively correlated with immune infiltration of CD8 ${ }^{+}$ T-cells, CD4 ${ }^{+}$T-cells, B-cells, neutrophils, macrophages and dendritic cells. Furthermore, CMTM2 has significantly positive correlations with the infiltrating levels of $\mathrm{CD} 8^{+} \mathrm{T}$-cells, $\mathrm{CD} 4^{+} \mathrm{T}$-cells, neutrophils, macrophages and dendritic cells in HCC patients. CMTM5 also has dramatically positive associations with the infiltrating levels of $\mathrm{CD} 8^{+}$T-cells and neutrophils in LIHC. However, there is no connection between CMTM8 and TIICs. 


\section{A potential mechanism underlying the effects of independent prognostic CMTM family genes on HCC Carcinogenesis}

We performed GSEA on differentially expressed CMTM family proteins with independent prognostic value to assess the potential biological mechanism by which differential expression of CKLF and CMTM7 affects the occurrence of liver cancer. The GSEA showed that CKLF might be related to biological processes, such as cell cycle, DNA replication, spliceosome and base excision repair (Fig. 9A). Moreover, the enrichment also indicated that act on the same biological effect with CKLF. Whereas CMTM7 may be involved in JAK-STAT, MAPK, B cell receptor, TOLL-like receptor, cancer, and WNT signaling pathways (Fig. 9B).

\section{Discussion}

The CMTM family has been found in a variety of cancers, including liver cancer. However, the mechanism in liver cancer has not been clarified. In this research, the mRNA expression, DNA methylation and prognostic value of $\mathrm{CmTM}$ family genes in $\mathrm{HCC}$ were investigated. We hope that our findings will provide novel diagnostic biomarkers and treatment designs for patients with HCC.

In the present study, all the CMTMs mRNA expressions in HCC were shown. Besides, the high level of CKLF and CMTM 1/4/7 were significantly related to worse survival outcomes in HCC patients, while CMTM2/ 5 was connected with better survival outcomes. Although CMTM3/6/8 wasn't significantly concerned with OS and PFS in HCC, $47 \mathrm{CpGs}$ of CMTMs demonstrated significant prognostic values. Furthermore, multivariate cox analysis indicated that CKLF and CMTM7 were independent high-risk prognostic factors for the unfavorable OS of HCC patients. Through pathway enrichment analysis, we figured out that the members of an independent prognostic value in CMTM family were involved in the immune process, tumor development via JAK-STAT and MAPKsignaling pathways. Moreover, we also studied the correlation of tumor immune infiltrating cells with CMTMs. We revealed that CKLF and CMTM1/3/6/7 were significantly associated with TIICs. However, there was no significant correlation between CMTM8 expression and TIICs.

Chemokine-like factor (CKLF), as the first discovered member of CMTM family, was cloned by suppression subtractive hybridization (SSH) technology 2001[14]. It has been reported that CKLF was downregulated in lung cancer tissues but upregulated in ovarian cancer tissues[15]. CKLF was overexpressed in HCC tissues and predicted poor survival and related to vascular invasion of HCC patients. Mechanistically, CKLF could trigger the IL-6/STAT3 signaling pathway to promote HCC proliferation and migration and inhibit the apoptosis induced by Doxorubicin[11]. Previous studies demonstrated that arterial injury-induced CKLF overexpression was positive associated with the accumulation of vascular smooth muscle cells (VSMC) and neointimal hyperplasia[16]. In vitro study indicated that reduction of CKLF1 in vascular smooth muscle cells (VSMC) could promote cell death at the G2/M phase of cell cycle progression through a PI3K/Akt/NF-KB dependent signal pathways[16]. It was found that CKLF1 was also overexpressed in some innate immune diseases, such as lupus nephritis 
(LN) and arthritis $[17,18]$. That the expression of CKLF could increase in activated CD4 ${ }^{+}$and CD $8^{+}$ lymphocytes[19]. In addition, CKLF modulates chemotactic activity and activates neutrophils through the MAPK signal pathway[20]. Similarly, our study showed that CKLF mRNA expression was upregulated in HCC tissues compared with non-cancer tissues, and its expression was significantly correlated with tumor stage, lymph node metastatic status, and tumor grade of patients. In terms of prognosis, high CKLF expression was related to poor OS and PFS of HCC patients, and six CpGs of CKLF were correlated with significant OS time in HCC patients. The results indicated that CKLF was an independent prognostic marker of HCC, and its deeper mechanism and specific function in HCC needed further research.

CMTM1 has been found in a variety of cancers, such as lung, breast, liver cancers, kidney and ovarian, and high level of CMTM1 promoted cell proliferation of breast cancer and inhibited TNF-a induced apoptosis by stimulating NF-KB pathway[21]. Additionally, NSCLC patients with higher CMTM1 expression had significantly higher pathological staging and a shorter prognosis of OS and PFS, suggesting that CMTM1 may contribute to the process of chemotherapy resistance[22]. A previous study showed that CMTM1 was overexpressed in lymphoma cells, and the injection of CMTM1 polypeptide in vitro can specifically promote apoptosis of lymphoma cells and may serve as a potential therapeutic for lymphoma treatment. Of note, mRNA expression of CMTM1 was remarkably associated with patients' family history and TNM stages of HCC patients[23]. Further analysis by $\mathrm{HHC}$ results indicated that negative expression of CMTM1 was related to poor prognosis of HCC patients. A similar tumorigenic effect of CMTM1 in HCC was also demonstrated in our present study[24]. Our dates found that mRNA expression of CMTM1 was up-regulated in HCC, and its expression was markedly associated with clinical features such as cancer stages, node metastasis status and tumor grades. Our data also revealed that CMTM1 prompts poor OS and PFS in HCC patients, and nine CpGs of CMTM1 were correlated with survival outcomes in patients of HCC. Given the specific studies on the role of CMTM1 in HCC remained unknown, CMTM1 is worthy of further exploration.

CMTM2 was higher expressed in diffuse-type gastric cancer and patients with shorter overall survival tended to express lower mRNA expression of CMTM2[25]. Previous research has shown that CMTM2 was reduced in HCC tissues and correlated with vascular invasion of HCC patients[26]. They further confirmed that CMTM2 expression was significantly associated with pathological grade and prognosis of HCC. Notably, down-regulation of CMTM2 can promote the Huh-7 and SMMC7721 cells' proliferation and migration via inducing the EMT (Epithelial-Mesenchymal Transition) process[26, 27]. What's more, the low expression of CMTM2 in metastatic salivary adenoid cystic carcinoma (SACC) in patients with tumor recurrence and perinerve infiltration suggests a significant role in the progression of tumor metastasis in SACC[28]. A study shows that intracellular CMTM2 can negative regulate HIV-1 transcription in U937 (human monocyte cell line) cells and Jurkat (lymphoblastoid T-cell line) through targeting the CREB and AP-1 pathways[29]. Likewise, our results revealed that CMTM2 was reduced in HCC tissues and the level of CMTM2 was significantly associated with patients' cancer stages. However, CMTM2 makes no difference with tumor grades and node metastasis status. Besides, high CMTM2 expression and one CpG of CMTM2 was related to favorable survival in HCC patients. We also found that CMTM2 was positive 
significantly correlated with the infiltrating levels of CD $8+T$ cells, CD4 + T cells, neutrophils cells, macrophages cells and dendritic cells. The specific role of CMTM2 in HCC deserves further study in order to deepen the understanding of HCC and develop new therapeutic methods.

CMTM3 was significantly lower in gastric cancer tissues than in normal tissues, and the expression of CMTM3 was significantly correlated with the stage and histological grade of gastric cancer. Moreover, increased expression of CMTM3 might be associated with a favorable prognosis in gastric cancer, and CMTM3 expression was an independent positive biomarker for gastric cancer[30]. Other studies have found that CMTM3 was obviously reduced in oral squamous cell carcinoma (OSCC), which was associated with the prognosis, recurrence and advanced TNM stage in patients with OSCC[31]. Additionally, the frequent DNA methylation in the CMTM3 gene promoter region was a potential unfavorable prognostic factor[32-34]. For instance, CMTM3 hypermethylation was associated with poor OS in male patients with laryngeal squamous cell carcinoma[33]. Previous research has shown that CMTM3 was found to be highly expressed in peripheral blood mononuclear cells (PBMCs), CD $4^{+} \mathrm{T}$ lymphocytes, resting $B$ lymphocytes and monocytes[35]. Concerning HCC research, compared with the control group (HL-7702 cell line), the expression level of CMTM3 in human liver cancer cell lines (Hep3B, HCCLM3, 97H and HepG2) was significantly down-regulated. Besides, CMTM3 prohibited proliferation and migration in HCC cells (HepG2) by inhibiting the JAK2/STAT3 signaling pathway[36]. In our present study, conflicting findings have been observed regarding the role of CMTM3 in HCC, and the mechanism of CMTM3 involved in the regulation of HCC development remains unknown. We found that CMTM3 was up-regulated in $\mathrm{HCC}$ and the mRNA expression of CMTM3 was markedly accord with the presence of cancer stages, node metastasis status and tumor grades of patients. However, CMTM3 was not apparently linked with the OS and PFS of HCC patients, three CpGs of CMTM3 were related to significant OS time. Therefore, the role of CMTM3 in HCC needs in-depth studies to elucidate.

CMTM4 was reported to be down-regulated in HCC compared with the adjacent non-cancer tissues. The expression of CMTM4 was negatively correlated with clinical (TNM) stage, tumor size and tumor metastasis rate, and plays an antitumor role in HCC[37]. Moreover, the expression of CMTM4 was significantly reduced in colorectal cancer. Further revealed that CMTM4 could significantly inhibit CRC cells growth in vivo experiments by inhibiting AKT, ERK1/2, and STAT3 signaling pathways[38]. It was reported that CMTM4 not only as a backup molecule modulating programmed cell death ligand 1 (PD-L1) protein but also identified as one of $\mathrm{CD}^{+} \mathrm{T}$ cell barren tumor-associated genes[39, 40]. Recent research demonstrated that CMTM4 was highly expressed in HCC in both TCGA dataset and Gene Expression Omnibus (GEO) database. CMTM4 was described as a prognostic marker of HCC, and CMTM4 was associated with the late clinical-stage, shorter OS, and PFS[41]. Also, the level of CMTM4 had negative correlations with influences immune cell infiltration (cytotoxic cells, CD8 + T cells, T cell and dendritic cells) in HCC tissues[41]. A similar tumorigenic role of CMTM4 in HCC was also displayed in our present study. CMTM4 was highly expressed in HCC tissues. The study also demonstrated that CMTM4 was markedly related to the patients' cancer stages, node metastasis status and tumor grades in patients with HCC. A correlation was identified between high CMTM4 expression and poor prognosis for PFS time, and 
8 CpGs of CMTM4 have also been found to be connected with survival outcome. Besides, we also found that CMTM4 was positively significantly correlated with the infiltrating levels of CD $4+T$ cells, B cells, neutrophils cells, macrophages cells and dendritic cells, indicating the role of CMTM4 in immunotherapy for HCC worthy further exploitation.

CMTM5 is closely $43 \%$ homology with CMTM3 and is involved in gene silencing. CMTM5 gene showed depressing tumor activities and was usually silenced by $\mathrm{CpG}$ methylation in various carcinomas, such as myeloid leukemia and breast cancer[12, 42, 43]. Studies have also reported a negative correlation between CMTM5 DNA promoter hypermethylation and its mRNA expression level in breast cancer[12]. Moreover, CMTM5 was obviously linked with differentiation, clinical staging, lymphatic metastasis and was predicted poor progression-free survival of patients in breast cancer in breast cancer[12, 44]. A casecontrol study observed a strong correlation between polymorphisms of rs3811178 in CMTM5 and HCC risk in the southern Chinese population[45]. Previous studies have found CMTM5 expressed at a low level in HCC. The low expression of CMTM5 is associated with advanced TNM stage, poor tumor differentiation and poor prognosis in HCC[46]. Similarly, another study proved that CMTM5 was lowly expressed in HCC tissues and cells. The level of CMTM5 can be inhibited after up-regulated microRNA$10 \mathrm{~b}-3 \mathrm{p}$ and then the proliferation, migration, and invasion abilities of HCC cells were suppressed in vitro [47]. Our study investigated that mRNA expression of CMTM5 was down-regulated in HCC tissues and CMTM5 expression was significantly associated with patients' tumor stages. High CMTM5 expression in HCC patients predicted better OS and two CpG of CMTM5 was related to overall survival time. Besides, CMTM5 expression was positively related to CD8 $+T$ cell and neutrophil. These findings are consistent with our studies and provide a foundation on which to perform further cell biological function assays and clinical sample validation in the future.

Recent research identified CMTM6 modulated antitumor immunity by maintaining the expression of programmed death-1 ligand 1(PD-L1)[48]. The higher expression of CMTM6 and PD-L1 in HCC, the worse the prognosis[49], but the better prognosis in colorectal cancer[50], and advanced-stage non-small cell lung cancer (NSCLC)[51]. Moreover, high CMTM6 levels also indicated that head and neck squamous cell carcinoma (HNSCC) [52], breast cancer[53], and gliomas with a bad prognosis[54], but predicted a favorable prognosis in lung adenocarcinoma[55] and hepatocellular carcinoma[56]. Besides, the high expression of CMTM6 in gliomas and malignant gliomas indicates a short survival time, which was involved in immune functions by regulation of T-lymphocyte cell-mediated anti-tumor immunity. In lung squamous carcinoma, CMTM6 expression was positively related to infiltration level of CD8 + T cell, neutrophil cell, macrophage cell and dendritic cell infiltration, and negatively correlated with infiltration of CD4 + T cell[57]. Another study has shown that Overexpression of CMTM6 could promote cancer cell proliferation, migration invasion by triggering epithelial-mesenchymal transition (EMT) in HCC, and a better prognosis of HCC was associated significantly with lower CMTM6 expression[58]. Furthermore, CMTM6 was found to be down-regulated in HCC tissues, and expression of CMTM6 was more likely correlated with alpha-fetoprotein (AFP), tumor staging, metastasis, and survival of HCC[56]. Likewise, our findings revealed that mRNA expression of CMTM6 was down-regulated in HCC tissues. The CMTM6 level was associated with patients' cancer prognosis. Although the expression of CMTM6 was not a 
statistical difference in OS and PFS, four CpG of CMTM6 was related to prognosis in patients with HCC. We also figured out that CMTM6 was positive significantly correlated with the infiltrating levels of CD $8+$ T cells, CD $4+T$ cells, B cells, neutrophils cells, macrophages cells and dendritic cells. Thus, CMTM6 is a potential target to improve the therapeutic of immune checkpoint inhibitors.

CMTM7 is down-regulated in gastric cancer, while over-expression of S0X10-dependent CMTM7 can impede the growing progress of cancer cells in gastric cancer[59]. Immunohistochemical experiments on 127 lung adenocarcinoma patients showed that CMTM7 was up-regulated (43/127), down-regulated (54/127), or maintained constant (30/127) in lung adenocarcinoma patients, and knockdown CMTM7 could result in a poorer survival outcome[60]. Moreover, Over-expression of CMTM7 could suppress the growth of cancer cells by triggering G1/S phase arrest and CMTM7 could promote EGFR internalization of repress the EGFR-mediated PI3K/AKT signaling pathway[61]. Additionally, studies have found that CMTM7 could decrease Rab5 activation by EGFR-AKT signaling pathway to promote non-small cell lung cancer (NSCLC) [62]. A report figured out that CMTM7 was obviously lower expressed in HCC tissues and the level of CMTM7 was negatively associated with TNM staging and tumor metastasis in HCC[63]. In addition, overexpression of CMTM7 can induce cell cycle arrest in G0/G1 phase by increasing p27 expression, decreasing cyclin D1 and cyclin dependent kinase 4/6 (CDK4/6) expression and inhibiting Akt signaling pathway. After that, the cell growth and tumorigenesis in HCC were obviously inhibited[63]. Interestingly, CMTM7 could not only link B-cell linker protein (BLNK) and B cell receptor (BCR) but also activation of downstream BCR signal transduction to MAPKs[64]. Previous studies have implicated that knockdown CMTM7 may promote cell death rate of B-1a (TrB-1a) cells[65]. So, CMTM7 exerts a pivotal role in BCR expression and survival of B-1a cells[66]. Contrary to the previous conclusion, CMTM7 mRNA was up-regulated in HCC in our research, higher mRNA expression of CMTM7 in HCC patients was associated with advanced grades or late stages. We demonstrated that high CMTM7 expression in patients with HCC means worse OS and PFS, and ten CpGs of CMTM7 were correlated with significant prognosis results in $\mathrm{HCC}$ patients, revealing that CMTM7 is also an independent prognostic indicator of HCC. To date, the expression of CMTM7 in HCC cell lines is still lack of fully explored. To explore the roles and specific mechanisms of CMTM7 in HCC and the development and progression of HCC needs further study.

As the database shows that CMTM8 was lower expression in bladder cancer and correlated with the progression of TNM stages. Overexpression of CMTM8 could suppress bladder cancer tissue cell growth, and possess a statistically significant predictor of better OS and PFS in bladder cancer patients[13, 67]. The functional study has shown that CMTM8 can promote cancer cells migration and invasion by reducing GSK3 $\beta$ to stimulate the $\beta$-catenin activation, while $\beta$-catenin knockdown suppressed these effects[68]. Moreover, the silence of CMTM8 in HepG2 cells could induce epithelial-to-mesenchymal transition-like processes through c-MET and ERK signaling pathways, indicating CMTM8 has an essential role in regulating HepG2 cells growth and invasion[69]. According to our research, we found that CMTM8 was up-regulated in HCC, and CMTM8 was closely correlated with patients' tumor grades. Besides, CMTM8 has no connection with survival (OS, PFS) in patients with HCC, but four CpG of CMTM8 was related to prognosis in patients with HCC. Up to now, specific studies on the effect of CMTM8 in HCC are 
still unclear. Therefore, further studies are still needed to clarify the molecular mechanism and exact role of CMTM8 in liver cancer carcinogenesis and progression.

\section{Conclusion}

Through investing mRNA expression, DNA methylation, we comprehensively described the prognostic value of CMTM family members in HCC and providing a valuable prospect foundation for the development of the CMTM family as diagnostic biomarkers of HCC. However, there are still some limitations that should be recognized in the study. Much of the research results come from public databases without the support of basic experiments. Thus, we need further experimental or clinical validation to confirm the effects of CMTMs in HCC.

To sum up, our results revealed that CKLF and CMTM1/3/4/7/8 are significantly highly expressed in HCC, and overexpression of six CMTM members was found to be positive relation with clinical tumor stages and cancer grades in HCC patients. Besides, CKLF and CMTM1/2/4/5/7 and CpGs methylation in all CMTM family members were markedly correlated with prognosis in patients with HCC. Multivariate cox analysis also demonstrated that high mRNA expressions of CKLF and CMTM7 were prognostic factors for shorter OS in HCC patients. Moreover, mRNA expression of CKLF and CMTM7 was dramatically associated with tumor immune infiltrating cells. These findings suggested that CKLF and CMTM7 can serve as prognostic biomarkers in HCC patients.

\section{Abbreviations}

CMTM, Chemokine-like factor (CKLF) MARVEL transmembrane domain-containing family; HCC, Hepatocellular carcinoma; OS, Overall survival; PFS, Progression-free survival; TCGA, The Cancer Genome Atlas; KM, Kaplan-Meier plotter; TIICs, Tumor immune infiltrating cells; TIMER2.0, Tumor immune Estimation Resource 2.0; GSEA,Gene set enrichment analysis; FDR, False discovery rate.

\section{Declarations}

\section{Acknowledgements}

We are grateful to all of the reviewers for their comments.

\section{Author contributions}

JB W conceived and designed the study. $\mathrm{D} \mathrm{L}$ and $\mathrm{SL} H$ were responsible for the collection and analysis of the reseach information. D L, LY S and JB W critically and carefully revised this manuscript. The authors read and approved the final manuscript.

\section{Funding}


This work was supported by the National Natural Science Foundation of China (Nos. 82060435), the Project of the Jiangxi Provincial Department of Science and Technology (Nos. 20202BAB206052).

\section{Availability of data and materials}

The data that support the findings of this study are openly available in The Cancer Genome Atlas (TCGA) program at https://portal.gdc.cancer.gov/; UALCAN at http://ualcan.path.uab.edu/; Kaplan-Meier plotter at http://kmplot.com/analysis/; MethSurv at https://biit.cs.ut.ee/methsurv/; Tumor immune Estimation Resource 2.0 at http://timer.cistrome.org.

\section{Declarations}

\section{Ethics approval and consent to participate}

There were no cell, tissue, or animal studies. No ethical requirements are involved.

\section{Consent for publication}

Not applicable.

\section{Consent for publication}

All authors agree to publish the paper.

\section{Competing interests}

The authors declare that they have no competing interests.

\section{References}

1. Sung H, Ferlay J, Siegel RL, et al.: Global cancer statistics 2020: GLOBOCAN estimates of incidence and mortality worldwide for 36 cancers in 185 countries. CA Cancer J Clin 2021.

2. Kulik L, El-Serag HB. Epidemiology and Management of Hepatocellular Carcinoma. GASTROENTEROLOGY. 2019;156(2):477-91.

3. Craig AJ, von Felden J, Garcia-Lezana T, et al. Tumour evolution in hepatocellular carcinoma. Nat Rev Gastroenterol Hepatol. 2020;17(3):139-52.

4. Han W, Ding P, Xu M, et al. Identification of eight genes encoding chemokine-like factor superfamily members 1-8 (CKLFSF1-8) by in silico cloning and experimental validation. GENOMICS. 2003;81(6):609-17.

5. Sánchez-Pulido L, Martín-Belmonte F, Valencia A, et al. MARVEL: a conserved domain involved in membrane apposition events. TRENDS BIOCHEM SCI. 2002;27(12):599-601.

6. Duan HJ, Li XY, Liu C, et al. Chemokine-like factor-like MARVEL transmembrane domain-containing family in autoimmune diseases. Chin Med J (Engl). 2020;133(8):951-8. 
7. Zhang JW, Liu TF, Chen XH, et al. Validation of aspirin response-related transcripts in patients with coronary artery disease and preliminary investigation on CMTM5 function. GENE. 2017;624:56-65.

8. Liu F, Liu X, Liu X, et al. Integrated Analyses of Phenotype and Quantitative Proteome of CMTM4 Deficient Mice Reveal Its Association with Male Fertility. MOL CELL PROTEOMICS. 2019;18(6):107084.

9. Wu J, Li L, Wu S, et al. CMTM family proteins 1-8: roles in cancer biological processes and potential clinical value. CANCER BIOL MED. 2020;17(3):528-42.

10. Li M, Luo F, Tian X, et al. Chemokine-Like Factor-Like MARVEL Transmembrane Domain-Containing Family in Hepatocellular Carcinoma: Latest Advances. FRONT ONCOL. 2020;10:595973.

11. Liu Y, Liu L, Zhou Y, et al. CKLF1 Enhances Inflammation-Mediated Carcinogenesis and Prevents Doxorubicin-Induced Apoptosis via IL6/STAT3 Signaling in HCC. CLIN CANCER RES. 2019;25(13):4141-54.

12. Zhou J, Lei J, Wang J, et al. Bioinformatics-Based Discovery of CKLF-Like MARVEL Transmembrane Member 5 as a Novel Biomarker for Breast Cancer. Front Cell Dev Biol. 2019;7:361.

13. Zhang $\mathrm{S}$, Pei $\mathrm{X}, \mathrm{Hu} \mathrm{H}$, et al. Functional characterization of the tumor suppressor CMTM 8 and its association with prognosis in bladder cancer. Tumour Biol. 2016;37(5):6217-25.

14. Han W, Lou Y, Tang J, et al. Molecular cloning and characterization of chemokine-like factor 1 (CKLF1), a novel human cytokine with unique structure and potential chemotactic activity. BIOCHEM J. 2001;357(Pt 1):127-35.

15. Cai X, Deng J, Ming Q, et al. Chemokine-like factor 1: A promising therapeutic target in human diseases. Exp Biol Med (Maywood). 2020;245(16):1518-28.

16. Duan Y, Zhang Y, Qu C, et al. CKLF1 aggravates neointimal hyperplasia by inhibiting apoptosis of vascular smooth muscle cells through PI3K/AKT/NF-KB signaling. BIOMED PHARMACOTHER. 2019;117:108986.

17. Ji Y, Zhang H, Yuan $\mathrm{H}$, et al. [Expression of chemokine like factor- 1 in nephridial tissue of lupus nephritis]. Zhong Nan Da Xue Xue Bao Yi Xue Ban. 2007;32(3):490-3.

18. Tao K, Tang X, Wang B, et al. Distinct expression of chemokine-like factor 1 in synovium of osteoarthritis, rheumatoid arthritis and ankylosing spondylitis. J Huazhong Univ Sci Technolog Med Sci. 2016;36(1):70-6.

19. Li T, Zhong J, Chen Y, et al. Expression of chemokine-like factor 1 is upregulated during $T$ lymphocyte activation. LIFE SCI. 2006;79(6):519-24.

20. Kong LL, Wang ZY, Han N, et al. Neutralization of chemokine-like factor 1, a novel C-C chemokine, protects against focal cerebral ischemia by inhibiting neutrophil infiltration via MAPK pathways in rats. J Neuroinflammation. 2014;11:112.

21. Wang J, Zhang G, Zhang Y, et al. CMTM1_v17 is a novel potential therapeutic target in breast cancer. ONCOL REP. 2014;32(5):1829-36. 
22. Si J, Zhang P, Tian D, et al. CMTM1_v17 is associated with chemotherapy resistance and poor prognosis in non-small cell lung cancer. WORLD J SURG ONCOL. 2017;15(1):34.

23. Cao L, Yang C, Zhu B, et al. A novel protein CMTM1-v5 specifically induced human lymphoma cells apoptosis in vitro and in vivo. EXP CELL RES. 2019;385(1):111623.

24. Song $X$, Zhang $S$, Tian $R$, et al. Expression and clinical significance of CMTM1 in hepatocellular carcinoma. Open Med (Wars). 2021;16(1):217-23.

25. Choi JH, Kim YB, Ahn JM, et al. Identification of genomic aberrations associated with lymph node metastasis in diffuse-type gastric cancer. EXP MOL MED. 2018;50(4):1-11.

26. Guo X, Zhang S, Tan S, et al. Downregulated CMTM2 Poses Potential Clinical Significance in Hepatocellular Carcinoma. DNA CELL BIOL. 2020;39(4):683-9.

27. Zhang S, Tian R, Bei C, et al. Down-Regulated CMTM2 Promotes Epithelial-Mesenchymal Transition in Hepatocellular Carcinoma. Onco Targets Ther. 2020;13:5731-41.

28. Mays AC, Feng X, Browne JD, et al. Chemokine and Chemokine Receptor Profiles in Metastatic Salivary Adenoid Cystic Carcinoma. ANTICANCER RES. 2016;36(8):4013-8.

29. Song HS, Shi S, Lu XZ, et al. Intracellular CMTM2 negatively regulates human immunodeficiency virus type-1 transcription through targeting the transcription factors AP-1 and CREB. Chin Med J (Engl). 2010;123(17):2440-5.

30. Su Y, Lin Y, Zhang L, et al. CMTM3 inhibits cell migration and invasion and correlates with favorable prognosis in gastric cancer. CANCER SCI. 2014;105(1):26-34.

31. Zhang H, Zhang J, Nan X, et al. CMTM3 inhibits cell growth and migration and predicts favorable survival in oral squamous cell carcinoma. Tumour Biol. 2015;36(10):7849-58.

32. Wang Y, Li J, Cui Y, et al. CMTM3, located at the critical tumor suppressor locus 16q22.1, is silenced by $\mathrm{CpG}$ methylation in carcinomas and inhibits tumor cell growth through inducing apoptosis. CANCER RES. 2009;69(12):5194-201.

33. Shen Z, Chen X, Li Q, et al. Elevated methylation of CMTM3 promoter in the male laryngeal squamous cell carcinoma patients. CLIN BIOCHEM. 2016;49(16-17):1278-82.

34. Li J, Chen C, Bi X, et al. DNA methylation of CMTM3, SSTR2, and MDFI genes in colorectal cancer. GENE. 2017;630:1-7.

35. Zhong J, Wang Y, Qiu X, et al. Characterization and expression profile of CMTM3/CKLFSF3. J Biochem Mol Biol. 2006;39(5):537-45.

36. Li W, Zhang S. CKLF-Like MARVEL Transmembrane Domain-Containing Member 3 (CMTM3) Inhibits the Proliferation and Tumorigenisis in Hepatocellular Carcinoma Cells. ONCOL RES. 2017;25(2):28593.

37. Bei C, Zhang Y, Wei R, et al. Clinical significance of CMTM4 expression in hepatocellular carcinoma. Onco Targets Ther. 2017;10:5439-43.

38. Xue $\mathrm{H}, \mathrm{Li}$ T, Wang P, et al. CMTM4 inhibits cell proliferation and migration via AKT, ERK1/2, and STAT3 pathway in colorectal cancer. Acta Biochim Biophys Sin (Shanghai). 2019;51(9):915-24. 
39. Mezzadra R, Sun C, Jae LT, et al. Identification of CMTM6 and CMTM4 as PD-L1 protein regulators. NATURE. 2017;549(7670):106-10.

40. Routh ED, Pullikuth AK, Jin G, et al. Transcriptomic Features of T Cell-Barren Tumors Are Conserved Across Diverse Tumor Types. FRONT IMMUNOL. 2020;11:57.

41. Zhou HQ, Li JH, Liu LW, et al. Increased CMTM4 mRNA expression predicts a poor prognosis in patients with hepatocellular carcinoma. Hepatobiliary Pancreat Dis Int. 2020;19(6):596-601.

42. Zhang H, Nan X, Li X, et al. CMTM5 exhibits tumor suppressor activity through promoter methylation in oral squamous cell carcinoma. Biochem Biophys Res Commun. 2014;447(2):304-10.

43. Niu J, Li H, Zhang Y, et al. Aberrant expression of CKLF-like MARVEL transmembrane member 5 (CMTM5) by promoter methylation in myeloid leukemia. Leuk Res. 2011;35(6):771-6.

44. Chen Z, Cui N, Zhao JS, et al.: Expressions of ZNF436, $\beta$-catenin, EGFR, and CMTM5 in breast cancer and their clinical significances. EUR J HISTOCHEM 2021, 65(1).

45. Bei C, Tan C, Zhu X, et al. Association Between Polymorphisms in CMTM Family Genes and Hepatocellular Carcinoma in Guangxi of China. DNA CELL BIOL. 2018;37(8):691-6.

46. Xu G, Dang C. CMTM5 is downregulated and suppresses tumour growth in hepatocellular carcinoma through regulating PI3K-AKT signalling. CANCER CELL INT. 2017;17:113.

47. Guan L, Ji D, Liang N, et al. Up-regulation of miR-10b-3p promotes the progression of hepatocellular carcinoma cells via targeting CMTM5. J CELL MOL MED. 2018;22(7):3434-41.

48. Burr ML, Sparbier CE, Chan YC, et al. CMTM6 maintains the expression of PD-L1 and regulates antitumour immunity. NATURE. 2017;549(7670):101-5.

49. Liu LL, Zhang SW, Chao X, et al. Coexpression of CMTM6 and PD-L1 as a predictor of poor prognosis in macrotrabecular-massive hepatocellular carcinoma. Cancer Immunol Immunother. 2021;70(2):417-29.

50. Peng QH, Wang $\mathrm{CH}$, Chen HM, et al.: CMTM6 and PD-L1 coexpression is associated with an active immune microenvironment and a favorable prognosis in colorectal cancer. J IMMUNOTHER CANCER $2021,9(2)$.

51. Zugazagoitia J, Liu Y, Toki M, et al. Quantitative Assessment of CMTM6 in the Tumor Microenvironment and Association with Response to PD-1 Pathway Blockade in Advanced-Stage Non-Small Cell Lung Cancer. J THORAC ONCOL. 2019;14(12):2084-96.

52. Chen L, Yang QC, Li YC, et al. Targeting CMTM6 Suppresses Stem Cell-Like Properties and Enhances Antitumor Immunity in Head and Neck Squamous Cell Carcinoma. CANCER IMMUNOL RES. 2020;8(2):179-91.

53. Tian Y, Sun X, Cheng G, et al. The association of CMTM6 expression with prognosis and PD-L1 expression in triple-negative breast cancer. Ann Transl Med. 2021;9(2):131.

54. Guan X, Zhang C, Zhao J, et al. CMTM6 overexpression is associated with molecular and clinical characteristics of malignancy and predicts poor prognosis in gliomas. EBIOMEDICINE. 2018;35:23343. 
55. Wang H, Gao J, Zhang R, et al. Molecular and immune characteristics for lung adenocarcinoma patients with CMTM6 overexpression. INT IMMUNOPHARMACOL. 2020;83:106478.

56. Zhu X, Qi G, Li C, et al. Expression and Clinical Significance of CMTM6 in Hepatocellular Carcinoma. DNA CELL BIOL. 2019;38(2):193-7.

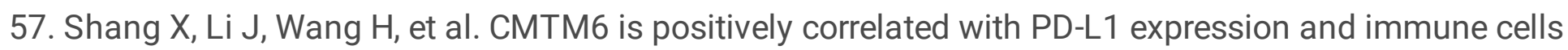
infiltration in lung squamous carcinoma. INT IMMUNOPHARMACOL. 2020;88:106864.

58. Huang $X$, Xiang $L$, Wang $B$, et al. CMTM6 promotes migration, invasion, and EMT by interacting with and stabilizing vimentin in hepatocellular carcinoma cells. J TRANSL MED. 2021;19(1):120.

59. Jin Y, Qin X, Jia G. SOX10-dependent CMTM7 expression inhibits cell proliferation and tumor growth in gastric carcinoma. Biochem Biophys Res Commun. 2018;507(1-4):91-9.

60. Liu Q, Su Y, Jiang GC, et al. Change of CMTM7 expression, a potential tumor suppressor, is associated with poor clinical outcome in human non-small cell lung cancer. Chin Med J (Engl). 2013;126(16):3006-12.

61. Li H, Li J, Su Y, et al. A novel 3p22.3 gene CMTM7 represses oncogenic EGFR signaling and inhibits cancer cell growth. ONCOGENE. 2014;33(24):3109-18.

62. Liu B, Su Y, Li T, et al. CMTM7 knockdown increases tumorigenicity of human non-small cell lung cancer cells and EGFR-AKT signaling by reducing Rab5 activation. Oncotarget. 2015;6(38):41092107.

63. Huang ZM, Li PL, Yang P, et al. Overexpression of CMTM7 inhibits cell growth and migration in liver cancer. KAOHSIUNG J MED SCI. 2019;35(6):332-40.

64. Miyazaki A, Yogosawa S, Murakami A, et al. Identification of CMTM7 as a transmembrane linker of BLNK and the B-cell receptor. PLOS ONE. 2012;7(2):e31829.

65. Liu Z, Liu Y, Li T, et al. Cmtm7 knockout inhibits B-1a cell development at the transitional (TrB-1a) stage. INT IMMUNOL. 2019;31(11):715-28.

66. Zhang Y, Wang JY, Han W. A role for CMTM7 in BCR expression and survival in B-1a but not B-2 cells. INT IMMUNOL. 2014;26(1):47-57.

67. Gao D, Hu H, Wang Y, et al. CMTM8 inhibits the carcinogenesis and progression of bladder cancer. ONCOL REP. 2015;34(6):2853-63.

68. Shi W, Zhang C, Ning Z, et al. CMTM8 as an LPA1-associated partner mediates lysophosphatidic acid-induced pancreatic cancer metastasis. Ann Transl Med. 2021;9(1):42.

69. Zhang W, Mendoza MC, Pei X, et al. Down-regulation of CMTM8 induces epithelial-to-mesenchymal transition-like changes via c-MET/extracellular signal-regulated kinase (ERK) signaling. J BIOL CHEM. 2012;287(15):11850-8.

\section{Figures}




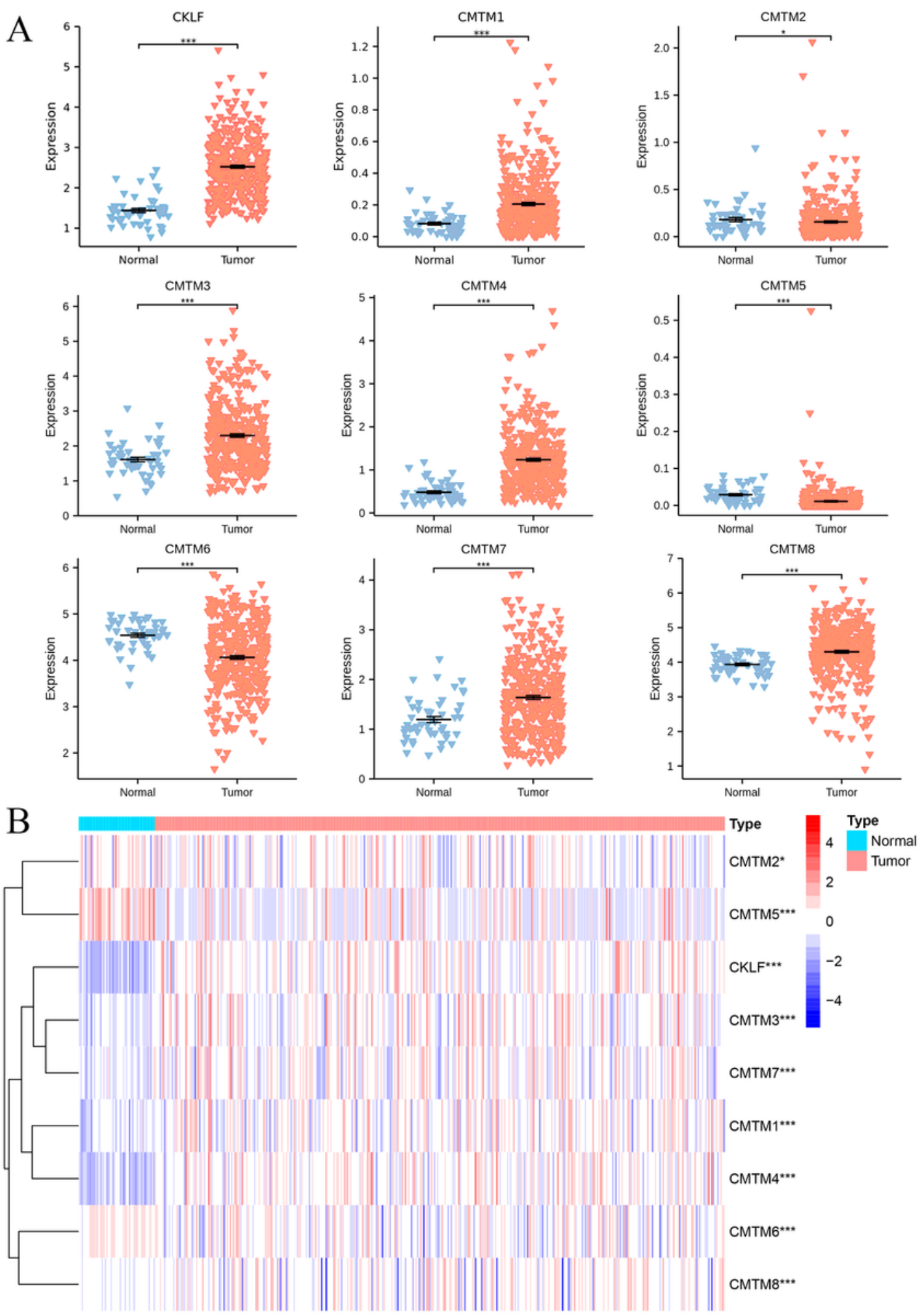

\section{Figure 1}

The mRNA expression profile of CMTM family members in HCC represented by scatter-plot (A), and a heat-map (B). * $p<0.05 ; * \star p<0.01 ; * \star * \quad p<0.001$. 

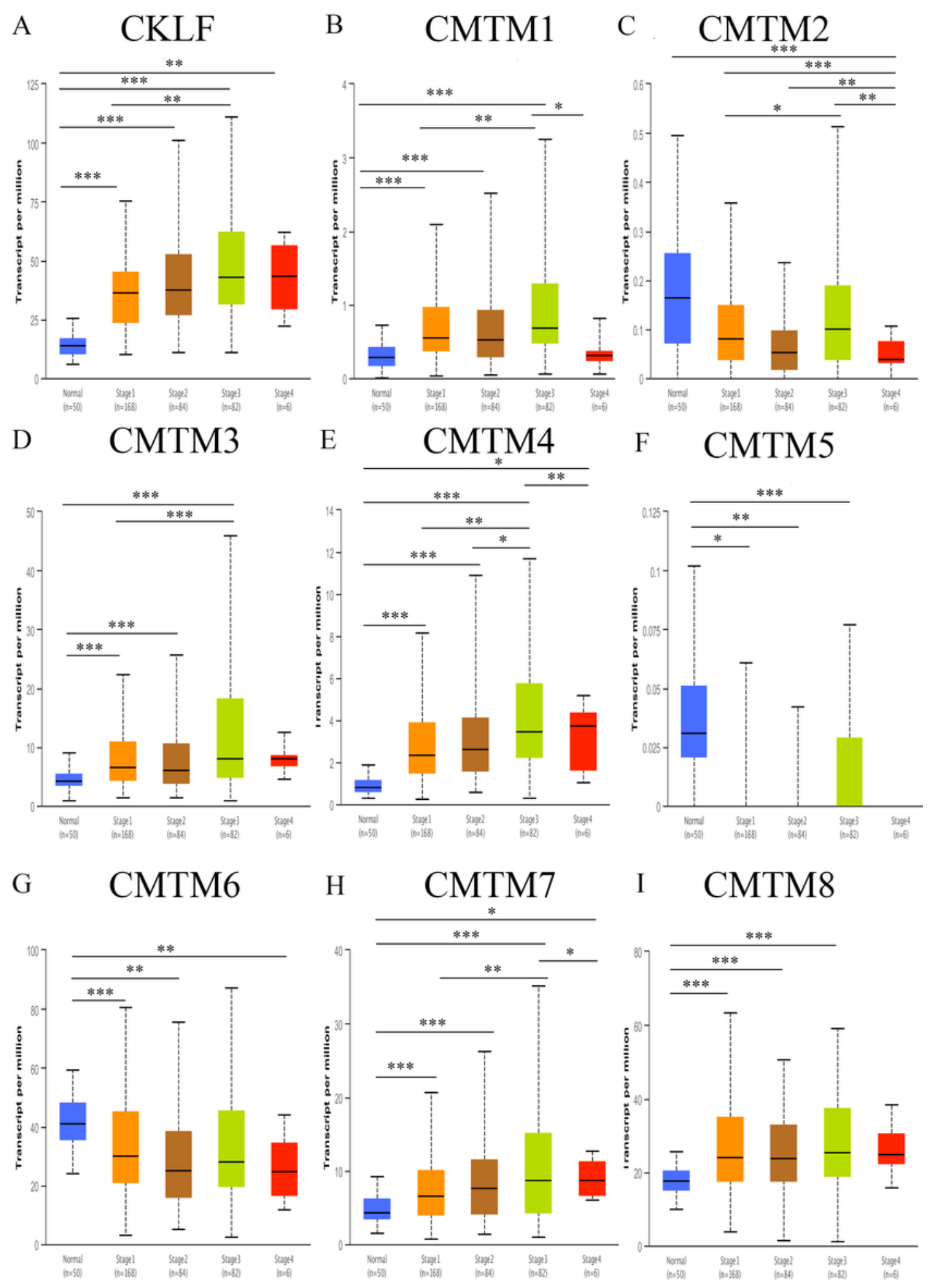

I CMTM8

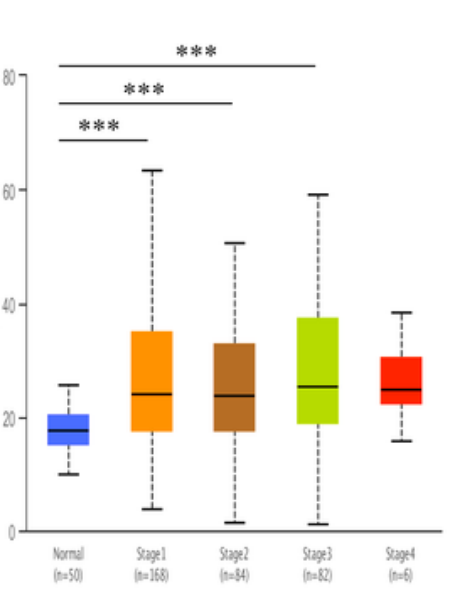

Figure 2

Relationship between CMTM family members and clinicopathological characteristics. Effect of CMTMs mRNA expression with tumor stages(A-I). * $p<0.05 ; * \star p<0.01 ; * \star \star p<0.001$. 


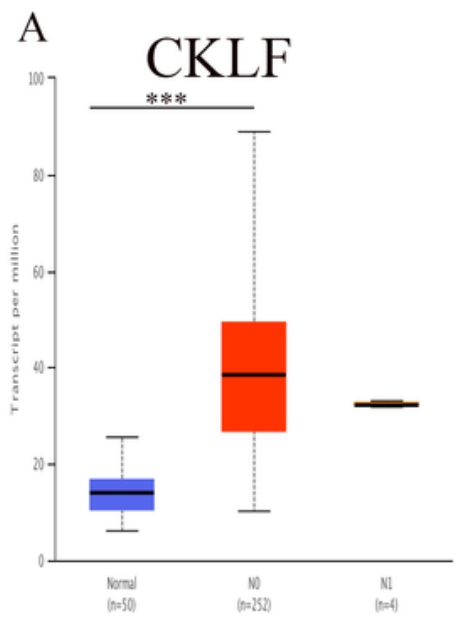

B

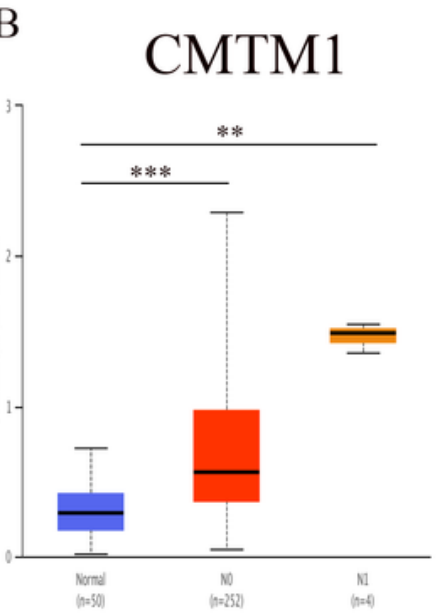

C CMTM2

D CMTM3

E $\quad$ CMTM4
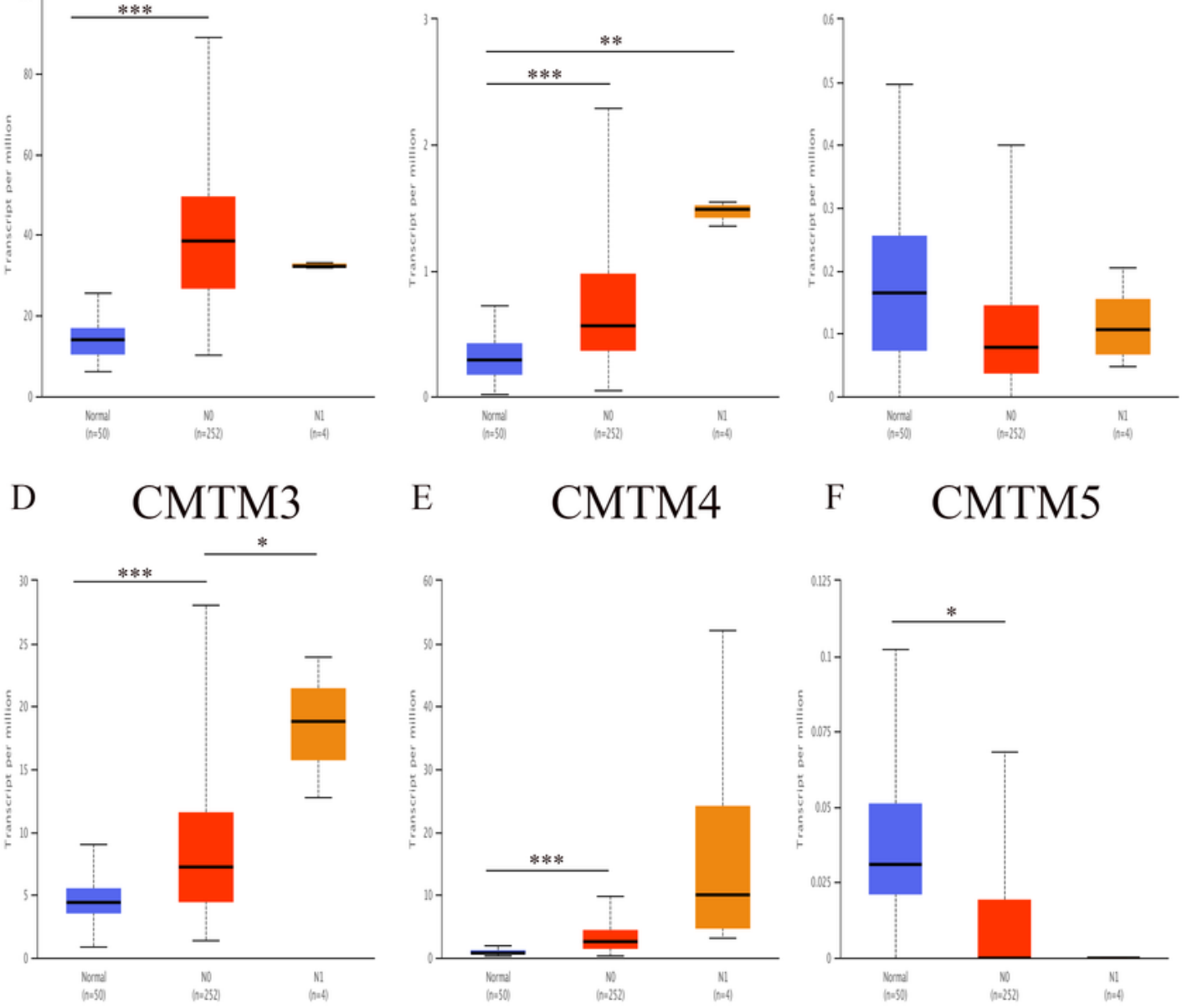

F CMTM5
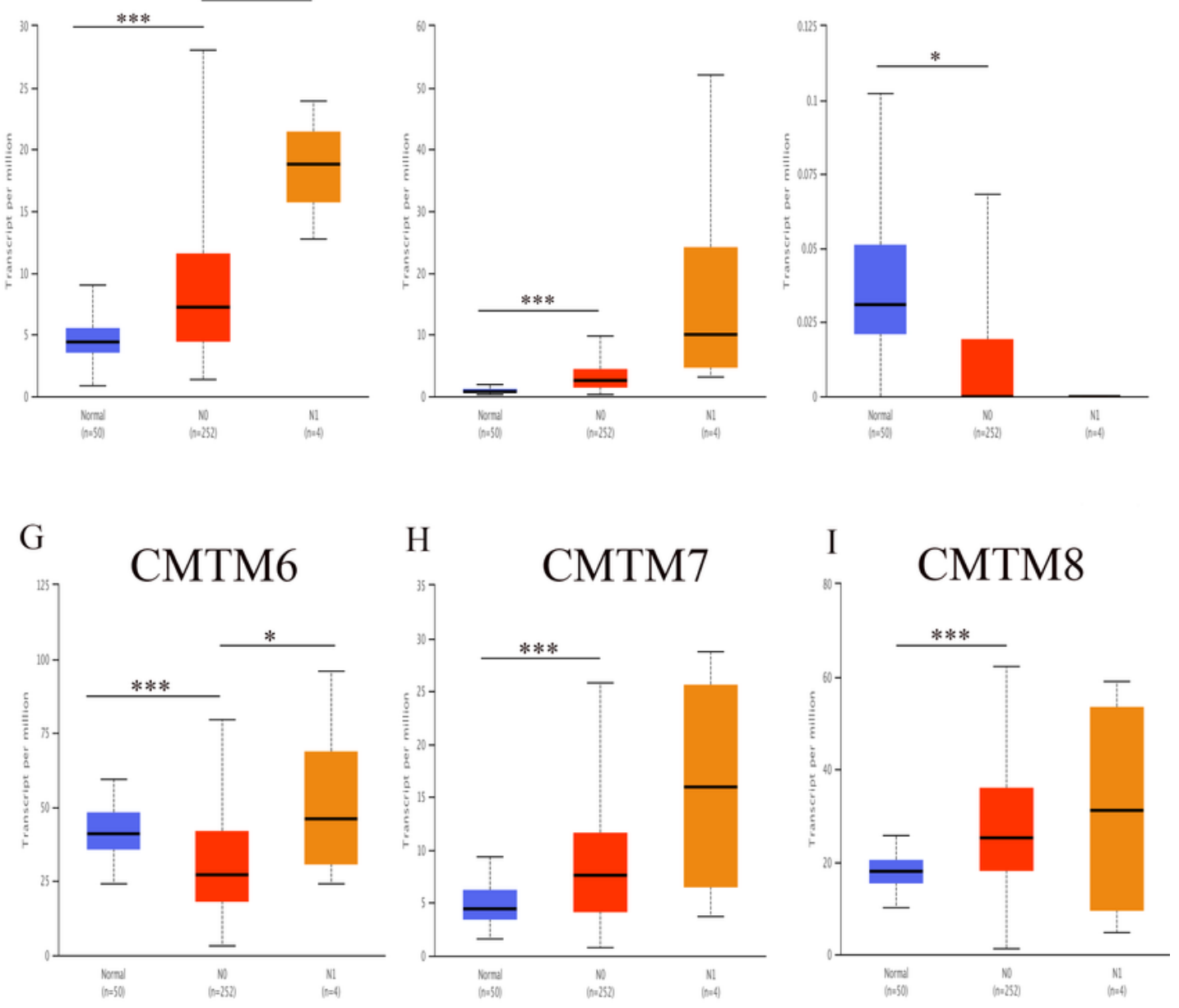

Figure 3

Relationship between CMTM family members and clinicopathological characteristics. Effect of CMTMs mRNA expression with node metastasis status $(A-I) .{ }^{*} p<0.05 ;{ }^{* \star} p<0.01 ; * \star \star p<0.001$. 

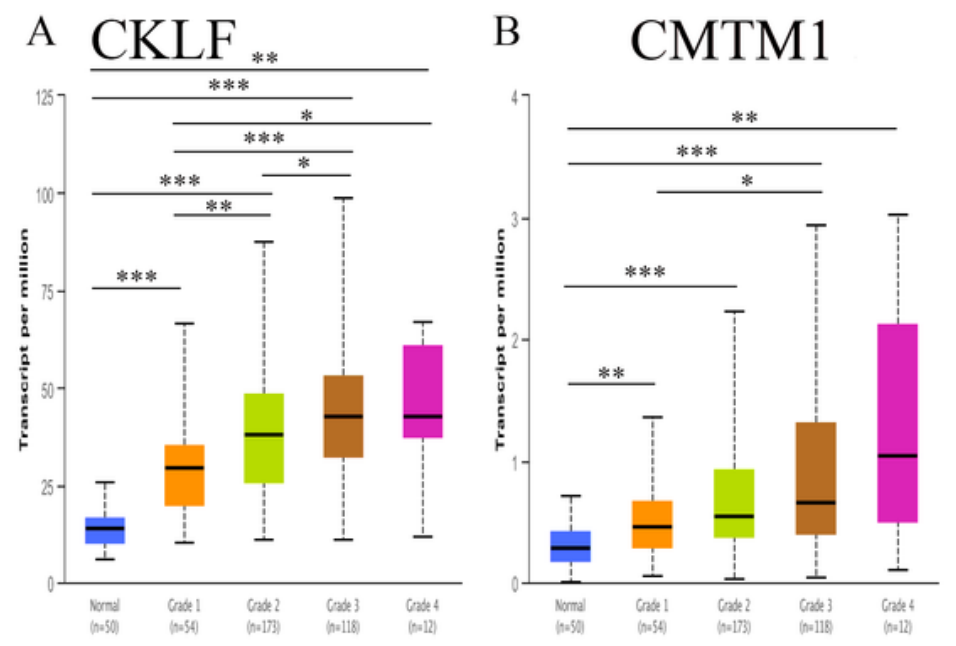

C. CMTM2

D CMTM3

$\mathrm{E}$
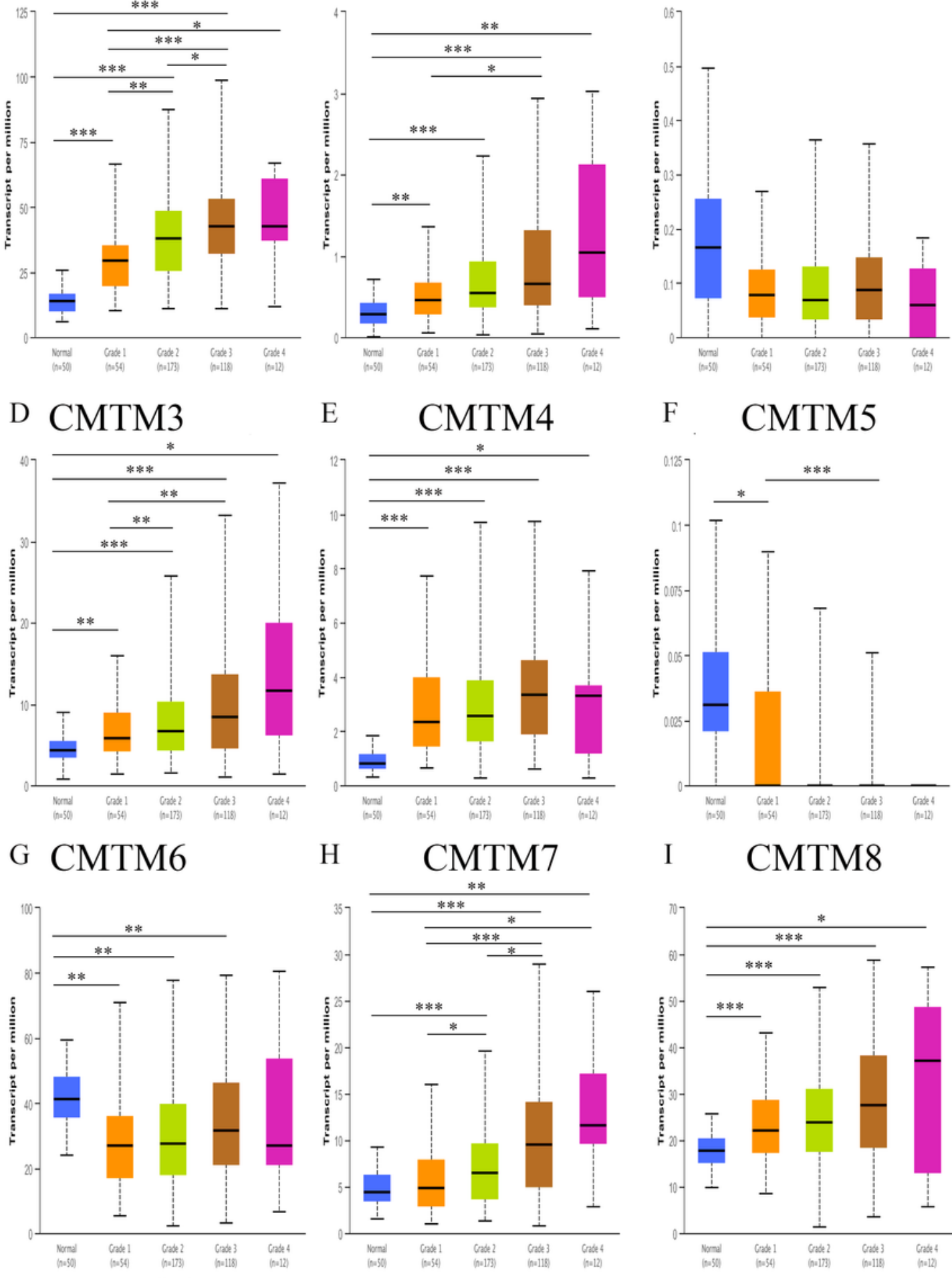

F CMTM5

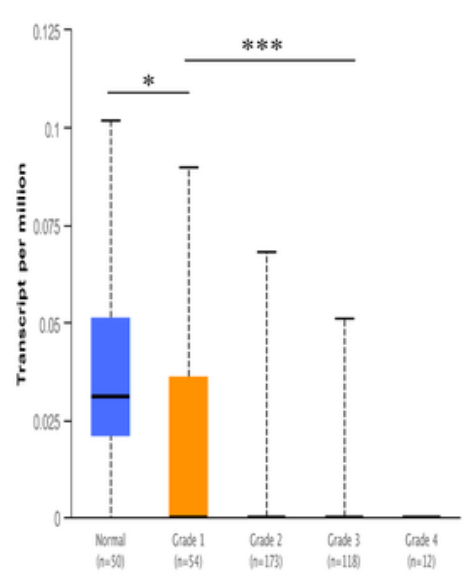

I $\quad$ CMTM8

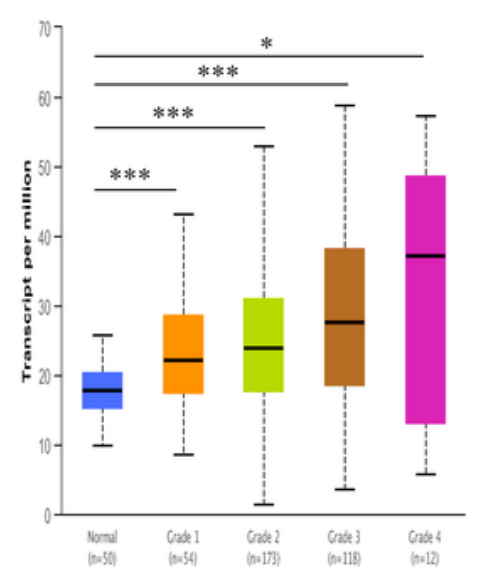

Figure 4

The CMTM family members are connected with clinicopathological characteristics. Effect of CMTMs mRNA expression with tumor grades $(A-I) .{ }^{*} p<0.05 ;{ }^{* \star} p<0.01 ;{ }^{* \star \star} p<0.001$. 

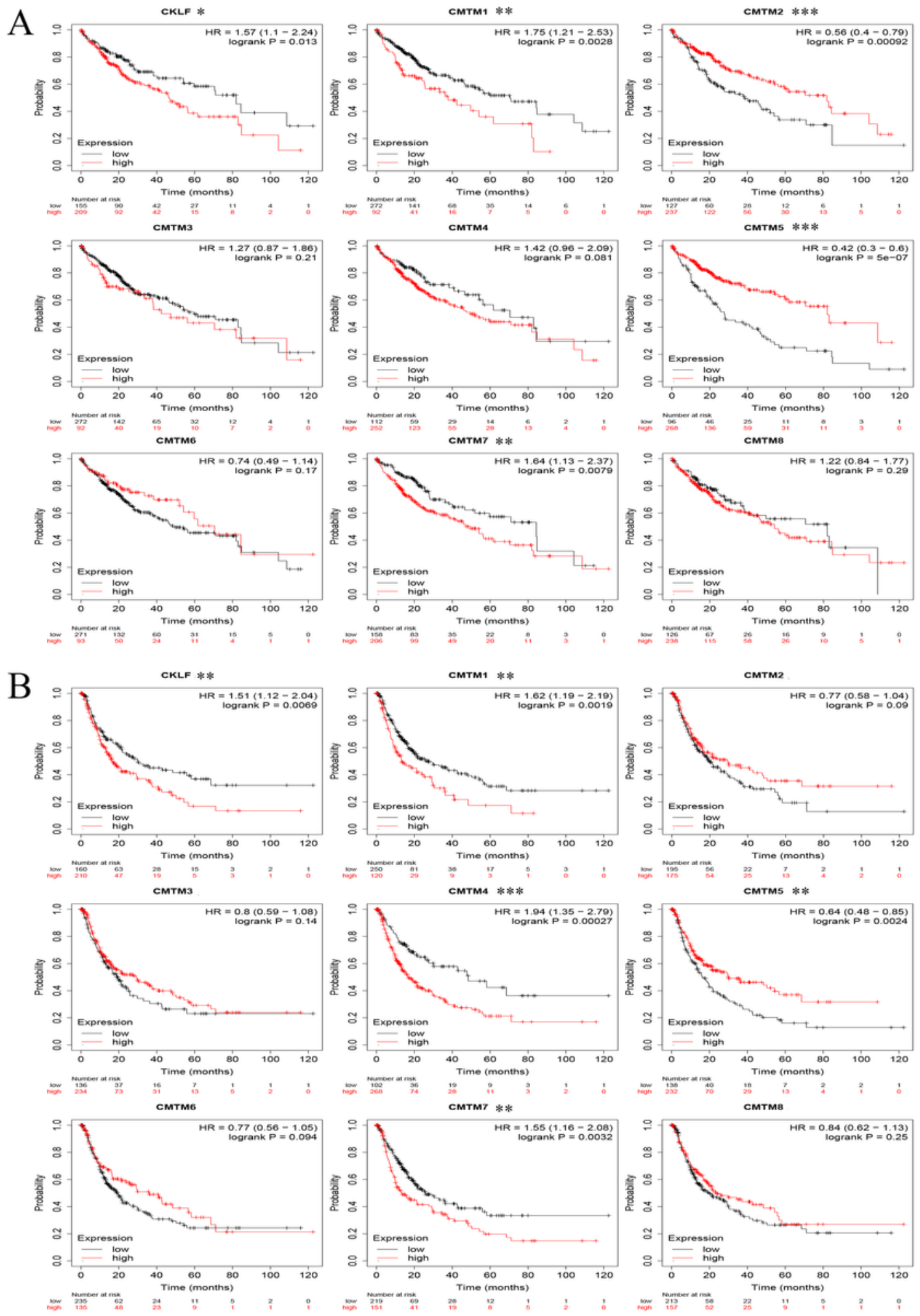

\section{Figure 5}

Prognostic value of mRNA expression levels of CMTM family members in patients with HCC (KaplanMeier plotter). Correlation of mRNA expression levels with OS(A) and PFS(B) in nine CmTM family members. ${ }^{*} p<0.05 ; * \star p<0.01 ; * \star \star p<0.001$. 

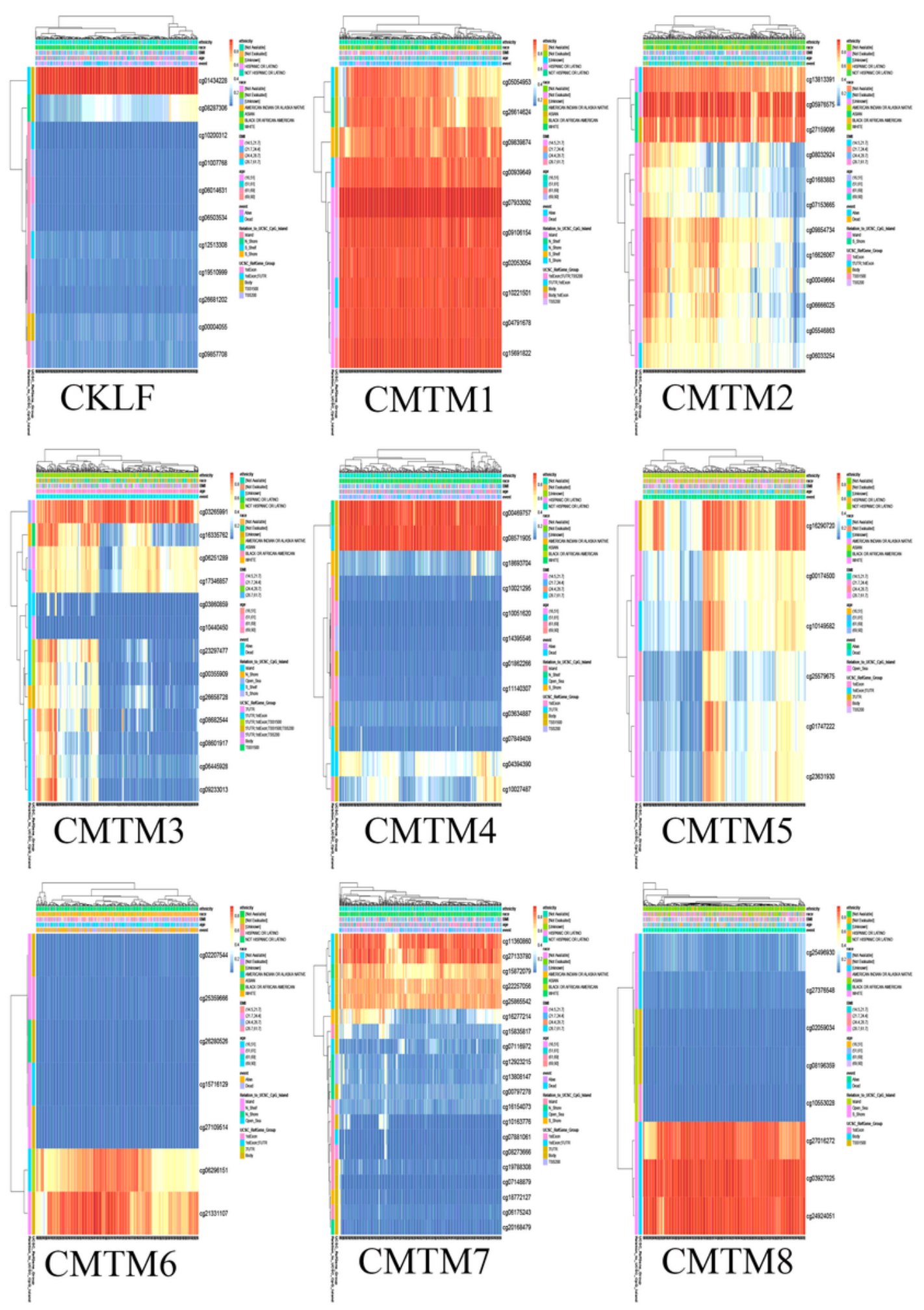

Figure 6

DNA methylation levels of CMTM family members in HCC (MethSurv database). Blue to red: low expression to high expression 


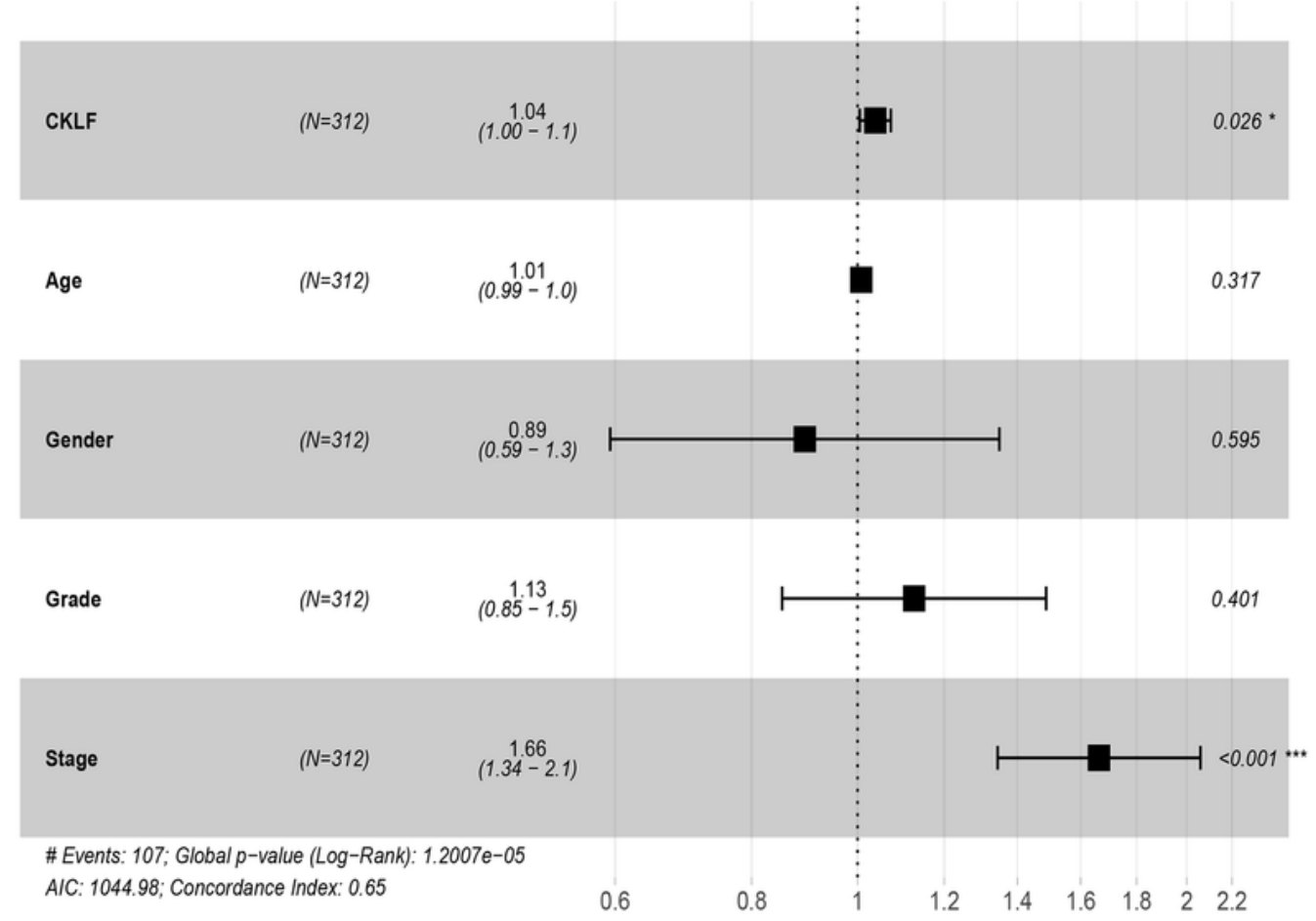

$\mathrm{B}$

Hazard ratio

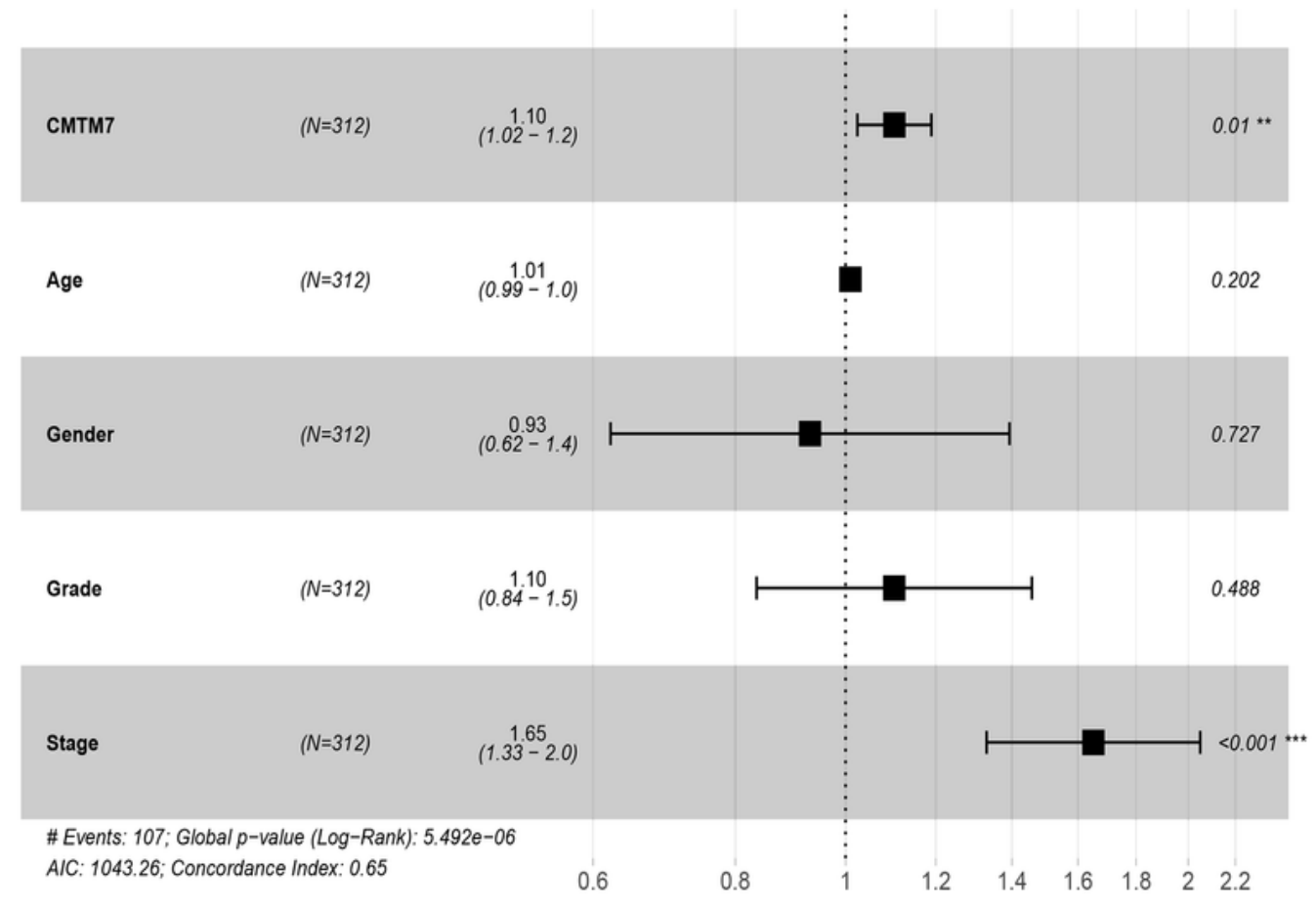

\section{Figure 7}

Forest plots of the results of multivariate Cox analyses of independent high-risk prognostic factor: $\operatorname{CKLF}(A)$ and CMTM7 (B). * $p<0.05 ; * \star p<0.01 ; * \star \star \star ~ p<0.001$. 

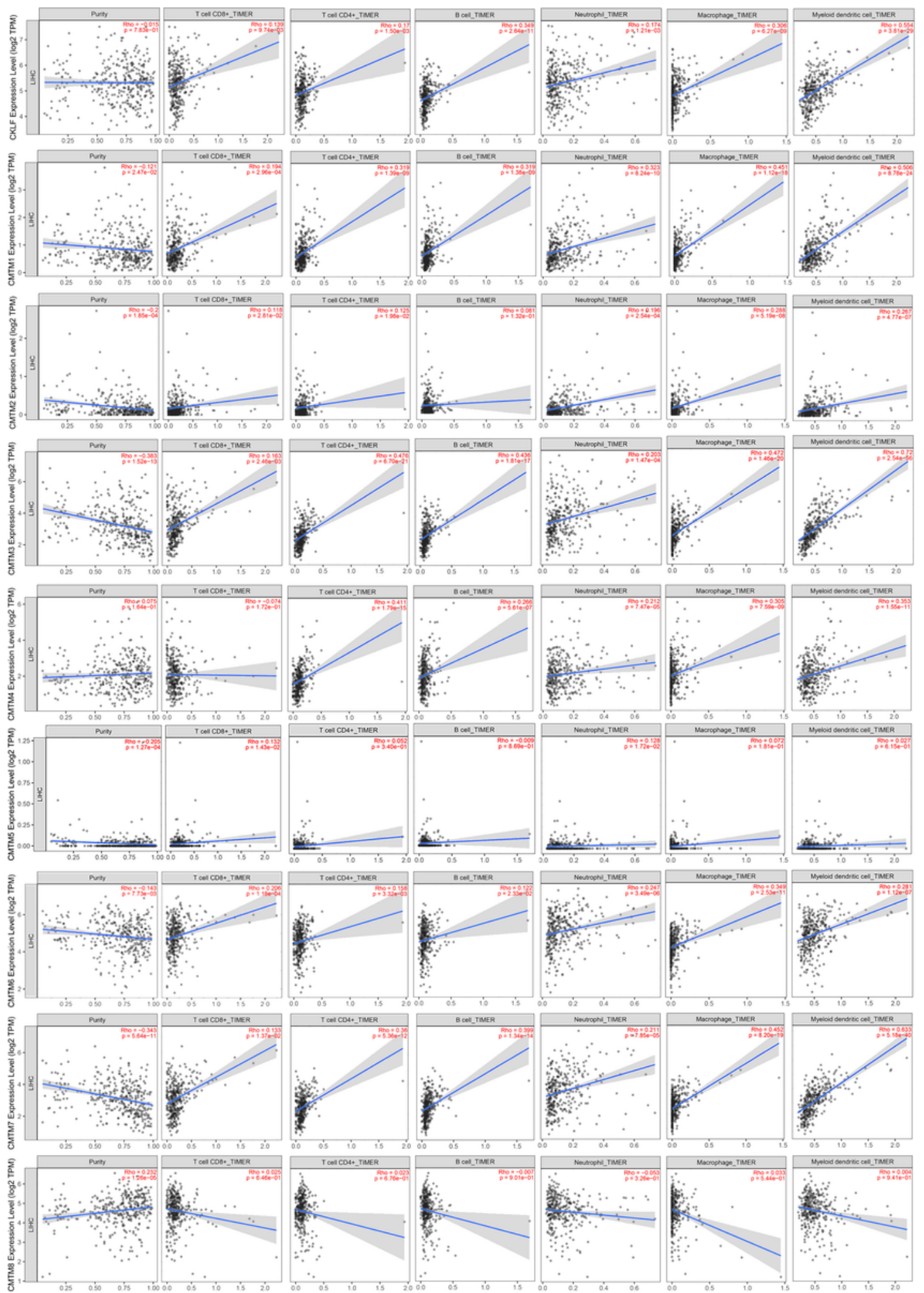

\section{Figure 8}

Correlation analysis of tumor infiltrating immune cells (TIICs) and members of the CMTM family. The leftmost panel shows the purity of the tumor. 
A

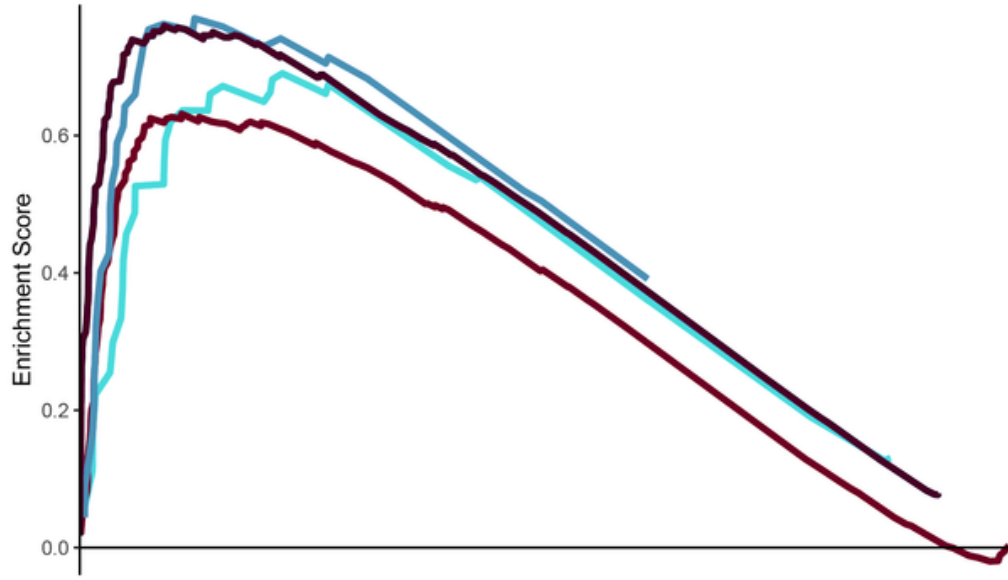

- KEGG_BASE_EXCISION_REPAIR

- KEGG_CELL_CYCLE

- KEGG_DNA_REPLICATION

- KEGG_SPLICEOSOME

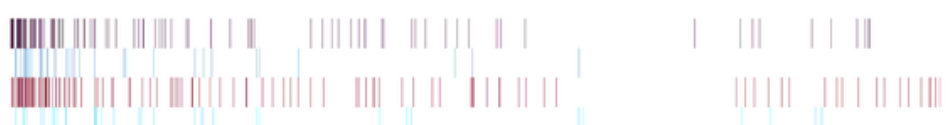

high expression<------------>low expression

B

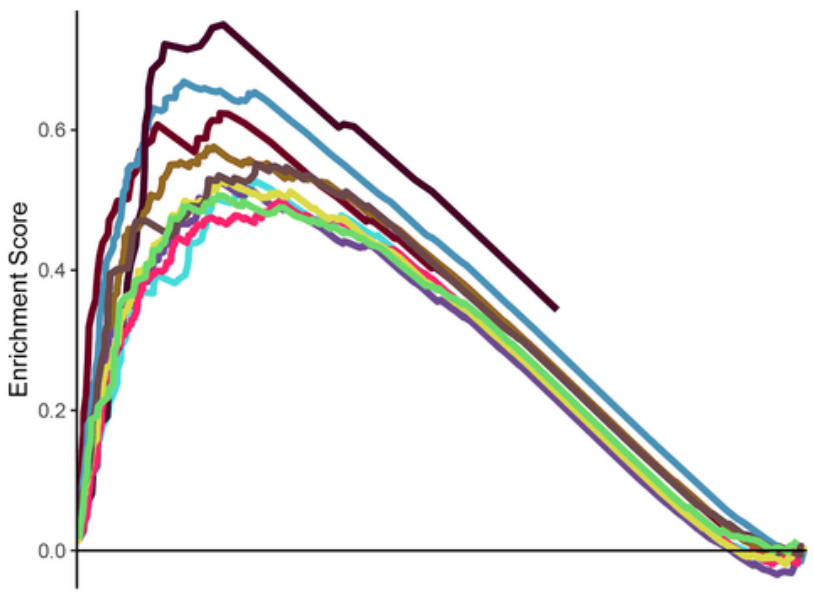

- KEGG_APOPTOSIS

- KEGG_B_CELL_RECEPTOR_SIGNALING_PATHWAY

- KEGG_CELL_CYCLE

- KEGg DNA REPLICATION

- KEGG_ENDOCYTOSIS

- KEGG_JAK STAT SIGNALING PATHWAY

- KEGG_MAPK_SIGNALING_PATHWAY

- KEGG_PATHWAYS_IN_CANCER

- KEGG_TOLL_LIKE_RECEPTOR_SIGNALING_PATHWAY

- KEGG_WNT_SIGNALING_PATHWAY

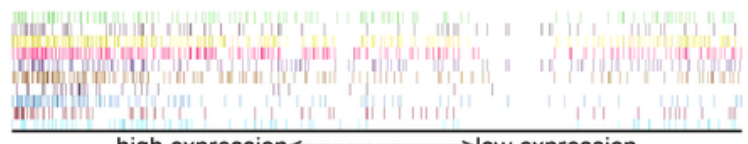

high expression<------------->low expression

\section{Figure 9}

A gene set enrichment analysis of CKLF (A) and CMTM7(B). 